\title{
Analysis of population-level determinants of legionellosis: spatial and geovisual methods for enhancing classification of high-risk areas
}

\author{
Jessie A. Gleason ${ }^{1 *}$, Kathleen M. Ross ${ }^{2,3}$ and Rebecca D. Greeley ${ }^{2}$
}

\begin{abstract}
Background: Although the incidence of legionellosis throughout North America and Europe continues to increase, public health investigations have not been able to identify a common exposure in most cases. Over $80 \%$ of cases are sporadic with no known source. To better understand the role of the macro-environment in legionellosis risk, a retrospective ecological study assessed associations between population-level measures of demographic, socioeconomic, and environmental factors and high-risk areas.

Methods: Geographic variability and clustering of legionellosis was explored in our study setting using the following methods: unadjusted and standardized incidence rate and SaTScan ${ }^{\mathrm{TM}}$ cluster detection methods using default scanning window of 1 and $50 \%$ as well as a reliability score methodology. Methods for classification of "high-risk" census tracts (areas roughly equivalent to a neighborhood with average population of 4000) for each of the spatial methods are presented. Univariate and multivariate logistic regression analyses were used to estimate associations with sociodemographic factors: population $\geq 65$ years of age, non-white race, Hispanic ethnicity, poverty, less than or some high school education; housing factors: housing vacant, renter-occupied, and built pre-1950 and pre-1970; and whether drinking water is groundwater or surface water source.
\end{abstract}

Results: Census tracts with high percentages of poverty, Hispanic population, and non-white population were more likely to be classified as high-risk for legionellosis versus a low-risk census tract. Vacant housing, renter-occupied housing, and homes built pre-1970 were also important positively associated risk factors. Drinking water source was not found to be associated with legionellosis incidence.

Discussion: Census tract level demographic, socioeconomic, and environmental characteristics are important risk factors of legionellosis and add to our understanding of the macro-environment for legionellosis occurrence. Our findings can be used by public health professionals to target disease prevention communication to vulnerable populations. Future studies are needed to explore the exact mechanisms by which these risk factors may influence legionellosis clustering.

Keywords: Legionellosis, Legionnaires' disease, Cluster analysis, GIS, SaTScan ${ }^{\mathrm{TM}}$, Ecological study

\section{Background}

Since the organism was first recognized during an outbreak in 1976, Legionella pneumophila has been

\footnotetext{
*Correspondence: Jessie.Gleason@doh.nj.gov

${ }^{1}$ Environmental and Occupational Health Surveillance Program,

New Jersey Department of Health, Trenton, NJ, USA

Full list of author information is available at the end of the article
}

identified as a relatively common cause of pneumonia in community and healthcare settings and has continued to garner attention globally [1]. Approximately 5000 cases of Legionnaires' disease are reported each year in the United States [2]; however, the actual number of hospitalized cases is estimated to be between 8000 and 18,000 [3] and the number of reported cases have been steadily 
increasing in the United States, Europe and Canada [4-7]. Although the first case of Legionnaire's Disease was recognized over 40 years ago, it remains a disease of emerging concern particularly in developed countries with more surveillance [1].

Legionellosis refers to two syndromes caused by bacteria of the genus Legionella including: (1) Legionnaires' disease, which is the more severe form of the infection and associated with pneumonia; and (2) Pontiac fever, which is milder and not associated with pneumonia [8]. Transmission to humans occurs through inhalation of aerosolized water that is contaminated with the bacteria. Legionellosis is not transmitted between individuals or by swallowing contaminated water; however, aspiration is also an important mode of disease transmission. Legionella species are common worldwide in the natural environment, including rivers, streams, and lakes and in artificial water environments and can survive in a range of environmental conditions, but grows best in warm temperatures between 32 and $42{ }^{\circ} \mathrm{C}(90-108 \mathrm{~F})$ [1].

The risk of contracting legionellosis is multifactorial, including factors which promote the proliferation of Legionella in the environment, host susceptibility factors, and exposure to aerosols. Known host susceptibility factors associated with the occurrence of legionellosis include older age ( $>50$ years), male sex, history of smoking, chronic lung disease, and poor immune function [9]. Outbreak investigations have identified the following environmental and exposure to aerosol risk factors: history of travel, residence in a healthcare facility, and proximity to water systems including: domestic water services (tanks, showers, faucets, stagnant warm pipes); cooling towers and evaporative condensers; whirlpool spas; respiratory therapy devices; vegetable misters; ice machines; and decorative fountains systems $[1,10,11]$. However, outbreaks of legionellosis account for only a minority of cases. Up to $80 \%$ of legionellosis cases are sporadic and public health investigations have not been able identify a common source, although these cases are not randomly distributed in time or space [12-14].

Research of population-level factors may be a useful complement to individual-level factor research which is the primary stream of research into causes and factors of legionellosis [15]. Some research has identified potential common population-level exposures including meteorological factors such as temperature, humidity, and water pressure [11, 16, 17], wastewater treatment plants [18], certain public water system characteristics [19], and other natural matrices and man-made water systems [11]; yet further investigation into population-level factors is needed to provide a better understanding of the complex nature of legionellosis. Although other studies of area-based predictors have assessed associations with infectious disease including giardiasis [20], salmonellosis [21], and campylobacteriosis [22], similar studies of legionellosis are limited.

Previous research has demonstrated methods for detection of small areas of excess incidence of Legionnaires' disease [23] as well as cluster detection as a prospective surveillance tool [24]. However, there is a paucity of ecological studies comparing legionellosis rates across geographic subsets (e.g., county, census tract) to estimate the impact of environmental and sociodemographic health disparities [25]. Incidence analyses are useful tools to describe and compare disease rates across geographic subsets. There is no steadfast rule to categorize incidence rates to identify areas of high occurrence and choice of cut point may lead to an over- or under- estimate of high occurrence areas. Use of spatial cluster analyses to identify areas of high occurrence can supplement findings from incidence rate analysis.

Studies which focus on spatial disease clustering may be successful at identifying macro-level factors, such as socioeconomic, demographic, and environmental factors, which contribute to the increase in disease [26]. SaTScan ${ }^{\mathrm{TM}}$ is a commonly used cluster detection software which calculates a spatial scan statistic by gradually scanning a window across space, noting the number of observed and expected observations inside the window at each location [27]. SaTScan ${ }^{\mathrm{TM}}$ users can adjust the size of the cluster scanning window (i.e., percent of the total population at risk); however, results can be misleading if the size of the cluster scanning window is made arbitrarily since a small scanning window can produce unstable clusters whereas a large window can overestimate the cluster area [28]. Researchers need to determine which model (e.g., maximum window size) best represents the true underlying clusters and how to quantitatively assess the fit of a model [29].

As increases in the incidence of legionellosis are observed, further investigation of population-level determinants of legionellosis is needed to expand our understanding of the complex mechanism of this disease. An ecological analysis was performed to estimate the association between legionellosis risk and the following population-level risk factors: age, race, ethnicity, poverty, education, housing factors including vacant, renter-occupied, and housing age, as well as drinking water source. To best classify census tracts at "high-risk" for legionellosis we explored the following spatial variability methods: (1) unadjusted and standardized incidence ratios across census tracts; (2) cluster detection with minimum and maximum window scanning and a reliability score method for classification of homogenous, reliable legionellosis clusters. The identification of geographic 
areas with high burden of disease and subsequently evaluating population-level determinants could allow for targeted public health interventions.

\section{Methods}

\section{Study population and design}

Our study setting was the state of New Jersey, the most densely populated of the 50 United States (U.S.), with a population of just under 9 million people. New Jersey is adjacent to New York City, New York the most populous city in the U.S., and Philadelphia, Pennsylvania the 5th most populous city. This statewide study setting provides a template for states/regions/countries to assess the role of population-level factors and legionellosis and not restrict research to urban city centers. Census tracts, areas roughly equivalent to a neighborhood with an average population of 4000 , were used as geographic unit of measure. Census tracts were developed and used by the U.S. Bureau of Census to collect, tabulate and present results of the decennial censuses. There are 2000 populated census tracts in New Jersey. Although U.S. census blocks, which contain about 1000 people, may provide finer scale geospatial analysis, data at that granular level is more limited and information for all factors of interest is not available.

A clinically compatible legionellosis case is classified as confirmed if it meets at least one of the confirmatory laboratory criteria: (1) culture isolation of any Legionella organism from respiratory secretions, lung tissue, pleural fluid, or other normally sterile fluid; (2) detection of $L$. pneumophila serogroup 1 antigen in urine [30]; or seroconversion of fourfold or greater rise in specific serum antibody titer to L. pneumophila serogroup 1 . In accordance with communicable disease reporting regulations set by the New Jersey Department of Health (NJDOH), healthcare providers must report diagnosed cases of legionellosis within $24 \mathrm{~h}$ of laboratory confirmation to the local health department where the case resides. Local health departments are responsible for initiating a public health investigation and subsequently reporting the case to the State health department through NJDOH's Communicable Disease Reporting and Surveillance System (CDRSS).

Confirmed legionellosis cases from 2003 to 2013 were extracted from CDRSS. Cases that were out-of-state during the entire incubation period (2-10 days prior to illness onset) were excluded from the study. Cases were also excluded if the CDRSS record did not contain age at onset, gender, date of symptom onset, or address. Legionellosis cases were geocoded to census tract based on their street address using ArcGIS version 10.2. Each census tract was assigned the aggregated count of legionellosis cases, the total 2010 U.S. Census population, and geographic coordinates of the census tract centroid for utilization in SaTScan ${ }^{\mathrm{TM}}$ software.

Census tract level demographic and socioeconomic variables of interest were obtained from the 2010 U.S. Census including percentages of total population $\geq 65$ years of age, non-white race, Hispanic ethnicity, below poverty, less than or some high school education. Housing variables included percentages of housing units which are vacant, renter-occupied, and built pre-1950 and pre-1970. Both pre-1950 and pre-1970 housing were explored in univariate analyses allowing for a simple comparison of effect estimate magnitude, but only pre-1950 was included in multivariate analyses. Each continuous exposure variable was categorized using the lower and upper quartiles rounded to the nearest $5 \%$ as cutoffs. The cutoffs, with distributions, for each variable can be found in Table 2. Additionally, each census tract population-weighted centroid was spatially joined to the public water system in which it fell. The primary drinking water source of each public water system was classified into ground water, surface water, and unknown.

\section{Spatial methods}

The following methods were used to explore geographic variability and clustering of legionellosis and to identify associations with population-level factors: (1) unadjusted and standardized incidence rate methods and (2) cluster detection using both 1 and 50\% window size sensitivity as well as a reliability score method to minimize window size sensitivity. Unadjusted legionellosis incidence rates were calculated for the 10-year study period for each census tract by dividing the number of confirmed legionellosis cases per census tract by total census tract population. Age- and sex-adjusted standardized incidence rates were calculated for the 10-year study period for each census tract using direct standardization. New Jersey's age- and sex-category population estimates available from the 2010 U.S. Census were used as the standard population. A choropleth map of the unadjusted and age- and sex-adjusted incidence rates was created using ArcGIS. Adjusted and unadjusted incidence rates were categorized into five intervals $(0,>0$ and $\leq 2 ;>2$ and $\leq 4 ;>4$ and $\leq 6$; and $>6$ ) for visualization.

\section{Cluster analysis}

Discrete Poisson models were used to identify retrospective, purely spatial clusters of legionellosis, using SaTS$\operatorname{can}^{\mathrm{TM}}$ software [31]. Standard clusters were defined as statistically significant clusters identified with SaTScan ${ }^{\mathrm{TM}}$ using the maximum scanning window size $(50 \%$ of the population at risk) and a minimum window size of $1 \%$. To mitigate SaTScan ${ }^{\mathrm{TM}}$ window size sensitivity and maximize the detection of stable legionellosis clusters, we 
calculated individual reliability scores for each census tract utilizing a methodology introduced by Chen et al. [28]. SaTScan ${ }^{\mathrm{TM}}$ was run 50 times adjusting the size of the cluster scanning window (1-50\%) by $1 \%$ with each run. Each SaTS $\operatorname{can}^{\text {TM }}$ run used a maximum likelihood function that identified the most likely cluster and secondary statistically significant clusters. $P$ values obtained through Monte Carlo hypothesis testing using 999 replications and relative risk values were assigned to each census tract.

Using the output from the $50 \mathrm{SaTScan}^{\mathrm{TM}}$ runs, reliability scores were calculated for each census tract as described in Chen et al. [28]. Reliability score is calculated by $R_{i}=C_{i} / S$, where $R_{i}$ is the reliability score for location $i, C_{i}$ is the number of scans for which that location $i$ is within a significant cluster, and $\mathrm{S}$ is the total number of SaTS$\mathrm{can}^{\mathrm{TM}}$ runs; and is defined as the likelihood that a census tract is reported within a significant cluster among systematically varying cluster scanning window sizes. Reliability scores can range from zero to one; zero indicates that the census tract was not identified within any statistically significant clusters in any SaTScan ${ }^{\mathrm{TM}}$ scans and one means that the census tract was identified within a statistically significant cluster in every SaTScan ${ }^{\mathrm{TM}}$ run.

Public health analyses routinely use choropleth maps to display incidence and cluster data, however Roth et al. [32] describe methods to improve the visualization of reliable, homogenous, and high-risk clusters. By utilizing this proposed methodology, reliability scores and relative risk values were concurrently illustrated with bivariate choropleth map using ArcGIS. This technique used a two-dimensional color and transparency legend (Fig. 3).

\section{Statistical analysis}

Univariate logistic regression modeling was used to explore associations between population-level factors and three types of legionellosis occurrence (i.e., census tract identified through conventional incidence rate; default cluster methods; and reliable, high-risk cluster methods) with demographic, socioeconomic, and environmental risk factors. Census tracts were categorized as high-risk if the incidence rate was $>2$ cases per 100,000 persons per year (incidence rate during 10-year study period was divided by 10 to estimate annual rate) or if the census tract was located within a statistically significant cluster detected by the default cluster detection methods. Census tracts with a reliability score $\geq 0.5$ and a $R R \geq 2$ were categorized as high-risk for cluster detection using the reliability score method. These classification criteria are presented in Table 1.

Multivariate analyses explored associations of demographic, socioeconomic, and environmental risk factors of reliable, high-risk cluster census tracts compared to low-risk census tracts ( $R S=0$ and $R R<2$ ) while simultaneously controlling for the other factors. Census tracts which were associated with a cluster but did not meet the criteria of a reliable, high-risk cluster were not included in the multivariate analyses. Pairwise correlations between each continuous exposure variable, precategorization, was examined. Collinearity diagnostics were assessed with an a priori cutoff of 30 for the condition indices (CI). Stepwise logistic regression was used to create a model that required a significance level of 0.05 to allow a variable into the model and a significance level of 0.05 to remain in the model. Forward and backward model selection resulted in the same final model; while the full model had the lowest AIC value. Results are reported as adjusted odds ratios with $95 \%$ confidence intervals.

\section{Results \\ Descriptive data}

From 2003 to 2013, 1634 reported legionellosis cases in New Jersey met the study's inclusion criteria. Pairwise Pearson correlation coefficients between continuous predictor factors identified only weak and very weak correlations $(r<0.39)$ except for a moderate positive correlation between education and non-white race $(r=0.42)$, poverty and Hispanic ethnicity $(r=0.49)$, and a strong a correlation between renter-occupied and some high school

Table 1 Methods use to assess spatial variability of legionellosis, classification criteria for high-risk census tracts, and corresponding number of census tracts, cases, and estimated population captured by each method

\begin{tabular}{lllcc}
\hline $\begin{array}{l}\text { Method of spatial } \\
\text { variability }\end{array}$ & “High-risk" classification & $\begin{array}{l}\text { \# of census tracts } \\
\text { identified as “high-risk” }\end{array}$ & $\begin{array}{l}\text { \# of cases residing } \\
\text { in “high-risk" census tract }\end{array}$ & $\begin{array}{l}\text { Estimated population } \\
\text { within “high-risk" } \\
\text { census tract }\end{array}$ \\
\hline Unadjusted IR & IR $\geq 2$ per 100,000 & 744 & 1407 & $3,126,058$ \\
Standardized IR & $\mid R \geq 2$ per 100,000 & 724 & 1376 & $3,151,260$ \\
$1 \%$ cluster detection & Detected within a cluster & 93 & 243 & 327,824 \\
$50 \%$ cluster detection & Detected within a cluster & 259 & 412 & 981,983 \\
Reliable cluster detection & RS $\geq 0.5$ and a RR $\geq 2$ & 136 & 397 & 507,694 \\
\hline
\end{tabular}


education $(\mathrm{r}=0.61)$ and renter-occupied and non-white race $(r=0.61)$.

\section{Incidence rate}

Although incidence rate methods included the largest number of cases, these methods also have a correspondingly large number of census tracts and population size which will likely result in dampened effect estimates when modeled (Table 1). Cluster analyses had fewer census tracts identified, with the reliability score method appearing to maximize the number of cases included against number of census tracts as compared to the default 1 and $50 \%$ cluster methods. Among the 2000 populated census tracts in New Jersey, 969 (49\%) tracts had at least one confirmed case of legionellosis between 2003 and 2013 (range 1-10 cases). The average 10-year unadjusted incidence rate was 19.5 cases per 100,000 persons (range $0-264$ ) and the average 10-year adjusted incidence rate was 20.8 cases per 100,000 persons (range 0-1694) (Fig. 1a, b).

\section{Cluster detection}

SaTScan ${ }^{\mathrm{TM}}$ cluster detection was performed with a minimum and maximum size scanning window of 1 and $50 \%$
(Fig. 2a, b). The $1 \%$ scanning window resulted in 11 statistically significant clusters comprised of 93 census tracts, while the scanning window of $50 \%$ resulted in six statistically significant clusters comprised of 259 census tracts. Alternatively, the methodology proposed by Chen et al. [28] identified $322(16 \%)$ census tracts as belonging within at least one statistically significant cluster, of which 136 census tracts met the high-risk definition (RS $\geq 0.5$ and $R R \geq 2$ ) (Fig. 3).

\section{Statistical analyses}

Unadjusted odds ratios and 95\% confidence intervals of associations with population-level risk factors highlights differences among spatial methods of legionellosis occurrence (i.e., conventional incidence rate; default cluster census tracts; reliable, high-risk cluster census tracts) (Table 2). Census tracts classified as high-risk through the incidence rate method were more likely to be categorized into the highest non-white population category (OR 1.67; 95\% CI 1.28, 2.17 ); percentage of poverty category (OR $1.49 ; 95 \% \mathrm{CI}$ $1.17,1.90)$; lower education level; and housing units that are renter- occupied or built pre-1950 or pre1970 as compared with census tracts with lower rates
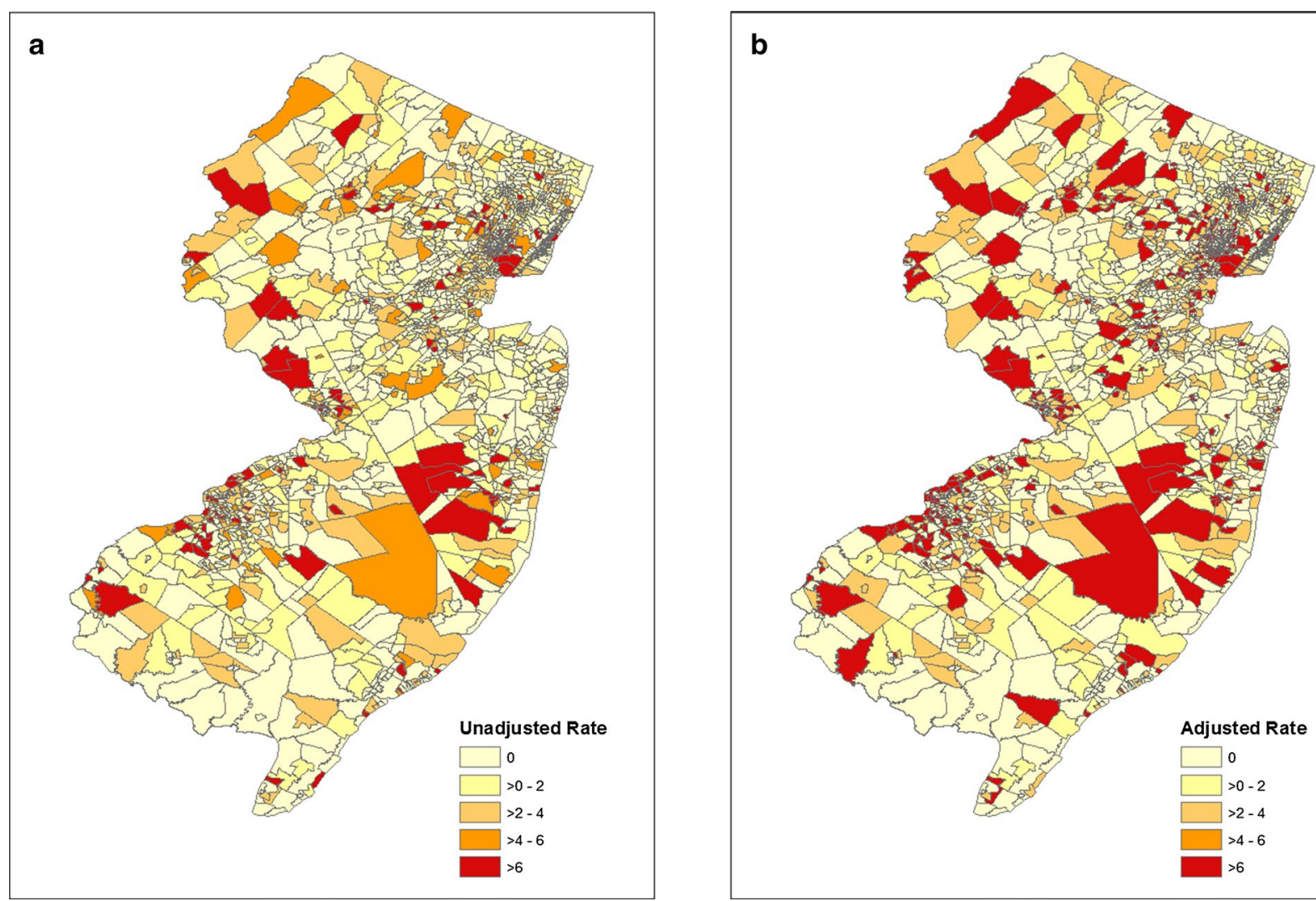

Fig. 1 Unadjusted and age and sex-adjusted legionellosis incidence rates by census tract in New Jersey, USA, 2003-2013 

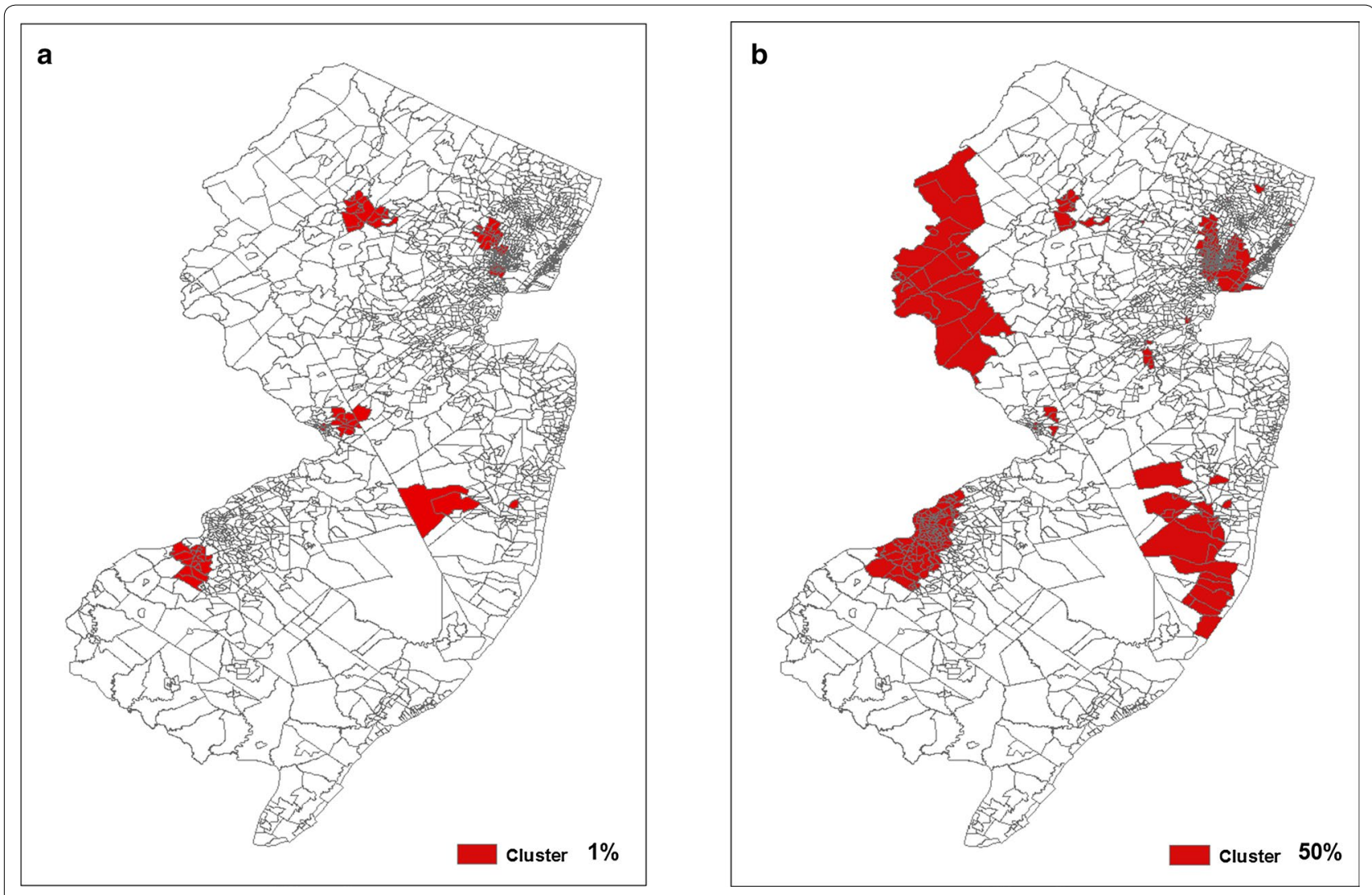

Fig. 2 Statistically significant legionellosis clusters detected using SaTScan ${ }^{\mathrm{TM}}$ software with census tract as geographic unit with 1 and $50 \%$ of the population at risk in New Jersey, USA, 2003-2013

of legionellosis. Comparatively, census tracts classified as high-risk through cluster analysis, both default and reliability score methods, found similar patterns of positive associations with population-level factors as those from incidence analysis methods, but effect estimates were much stronger for all factors explored. For example, census tracts classified as high-risk for incidence analysis were approximately 1.5 times more likely to be categorized into high poverty than lower risk census tracts compared with 3.8 times more likely that cluster-based high-risk census tracts would be categorized as high poverty. Cluster methods also found statistically significant associations with older age and vacant housing. Only minor, non-significant, differences were noted between findings from default cluster analyses and reliability score methods. The final multivariate logistic regression model found that census tracts with the highest percentages of older age (OR 0.38; 95\% CI 0.22, 0.67); Hispanic populations (OR 0.10; $95 \%$ CI 0.05 , 0.19 ); poverty (OR 7.21; 95\% CI 4.04, 12.86); and housing units built pre-1950 (OR 5.69; 95\% CI 2.82, 11.50) were positively statistically significantly associated with reliable, high-risk legionellosis cluster areas (Table 3). It is important to note that once other factors were controlled for, Hispanic ethnicity had a negative, statistically significant association.

\section{Discussion}

Our study assessed the relationship between populationlevel factors and areas of higher legionellosis occurrence. Census tracts classified as high-risk for legionellosis were more likely to have a high percentage of non-white population, poverty; low education level; and high percentage of housing units that are renter- occupied or built pre-1950 or pre-1970. Following adjustment of covariates, high percentage of older age, poverty, and housing units built pre- 1950 were positively associated while high percentage of Hispanic populations was negatively associated. Cluster detection methods for classifying highrisk census tracts were preferred from the incidence rate method.

\section{Spatial methods}

Little guidance exists for determining cut-off selection for incidence rates to classify high-risk census tracts. In 2009, the crude U.S. national incidence rate 


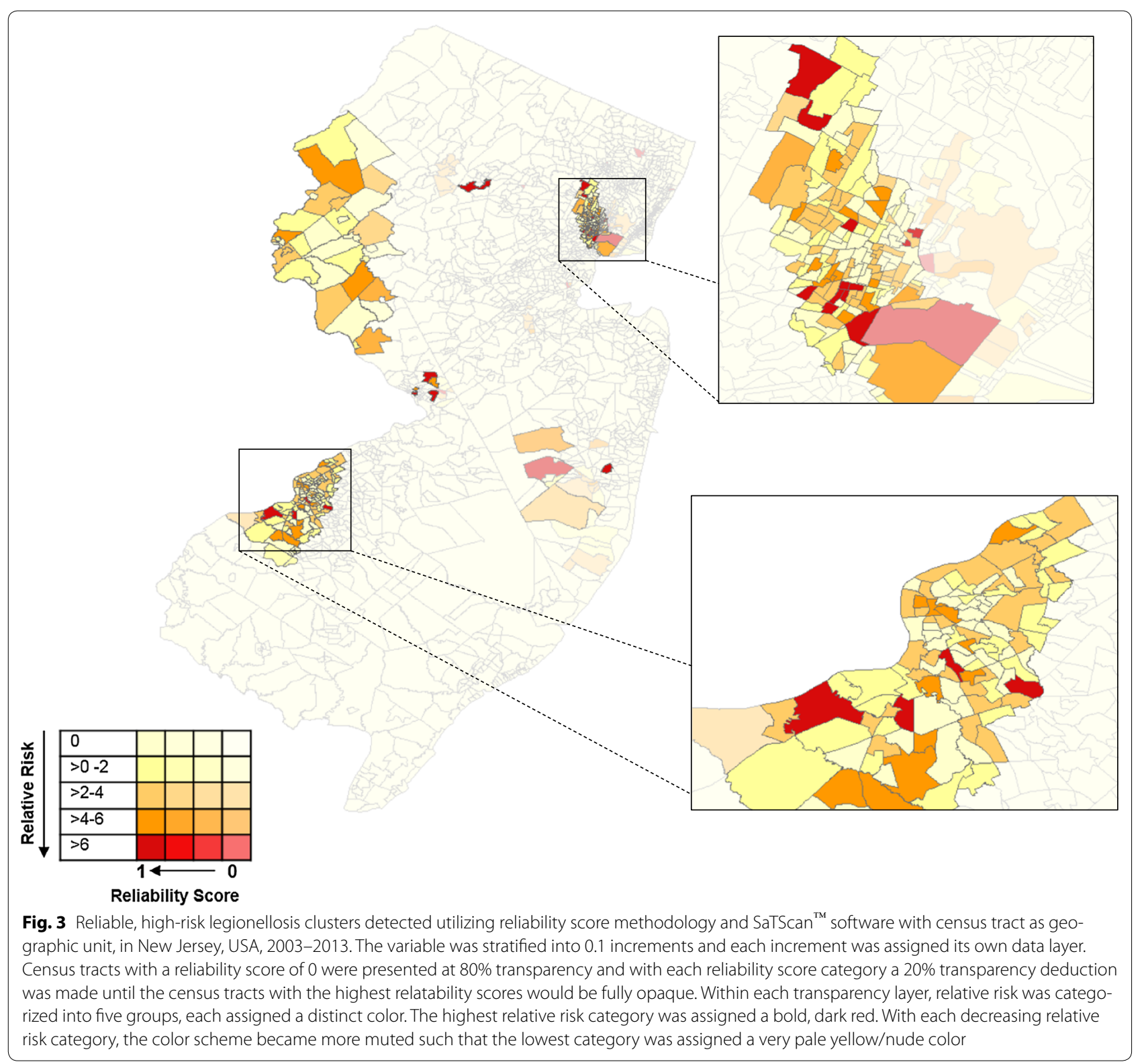

for legionellosis was 1.15 per 100,000 persons, the ageadjusted was 1.08 per 100,000 persons [33]. Therefore, a priori, study investigators selected an IR $>2$ (or IR $>20$ for 10-year rate) as a "high-risk" cut-off; which appears to have over-selected for high-risk census tract, with almost a third of the state's population included in a high-risk census tract. In general, our findings suggest that the potential over-selection led to limited ability of both univariate and multivariate models to either detect significant associations or resulted in more moderated effect estimates as compared with the cluster-based methodologies. Population-level analyses are important for contributing to our understanding of the macro-level determinants of legionellosis, and these analyses require accurate identification of at-risk areas.

Comparatively, results from incidence analyses and cluster detection methods supported each other; yet cluster detection methods, both default and reliability score methods, detected much stronger associations for all factors explored. However, we found that results did not meaningfully differ based on cluster detection method and default window scanning sizes are effective in estimating associations. Public health authorities can apply Chen's methodology for retrospective surveillance to identify homogenous, reliable, high-risk disease clusters for prioritizing targeted prevention outreach. 
Table 2 Unadjusted odds ratios and $95 \%$ confidence intervals of macro-level demographic and environmental factors and increased odds of legionellosis occurrence

\begin{tabular}{|c|c|c|c|c|c|c|}
\hline & \multicolumn{2}{|c|}{ Incidence rate ${ }^{a}$} & \multicolumn{2}{|c|}{ Standard cluster ${ }^{\mathbf{b}}$} & \multicolumn{2}{|c|}{ Reliable, high-risk cluster ${ }^{c}$} \\
\hline & OR & $95 \% \mathrm{Cl}$ & OR & $95 \% \mathrm{Cl}$ & OR & $95 \% \mathrm{Cl}$ \\
\hline \multicolumn{7}{|c|}{$\% \geq 65$ years of age } \\
\hline$<10$ & - & & - & & - & \\
\hline$\geq 10$ to $<15$ & 1.10 & $0.88-1.37$ & 0.60 & $0.44-0.81$ & 0.45 & $0.30-0.67$ \\
\hline$\geq 15$ & 0.84 & $0.66-1.07$ & 0.49 & $0.35-0.69$ & 0.33 & $0.20-0.53$ \\
\hline \multicolumn{7}{|c|}{$\%$ non-white race } \\
\hline$<10$ & - & & - & & - & \\
\hline$\geq 10$ to $<50$ & 1.00 & $0.79-1.26$ & 0.81 & $0.57-1.17$ & 0.89 & $0.54-1.47$ \\
\hline$\geq 50$ & 1.67 & $1.28-2.17$ & 2.67 & $1.86-3.83$ & 3.07 & $1.87-5.02$ \\
\hline \multicolumn{7}{|c|}{$\%$ hispanic ethnicity } \\
\hline$<5$ & - & & - & & - & \\
\hline$\geq 5$ to $<20$ & 1.19 & $0.96-1.48$ & 0.66 & $0.49-0.88$ & 1.05 & $0.70-1.58$ \\
\hline$\geq 20$ & 1.08 & $0.84-1.39$ & 0.47 & $0.32-0.67$ & 0.78 & $0.47-1.28$ \\
\hline \multicolumn{7}{|l|}{$\%$ poverty } \\
\hline$<5$ & - & & - & & - & \\
\hline$\geq 5$ to $<15$ & 1.34 & $1.09-1.65$ & 1.87 & $1.34-2.62$ & 1.40 & $0.86-2.25$ \\
\hline$\geq 15$ & 1.49 & $1.17-1.90$ & 3.78 & $2.68-5.32$ & 5.04 & $3.24-7.84$ \\
\hline \multicolumn{7}{|c|}{$\%$ less or some high school } \\
\hline$<5$ & - & & - & & - & \\
\hline$\geq 5$ to $<10$ & 1.24 & $0.99-1.55$ & 1.22 & $0.85-1.75$ & 1.52 & $0.92-2.48$ \\
\hline$\geq 10$ & 1.30 & $1.04-1.62$ & 2.35 & $1.70-3.25$ & 2.70 & $1.72-4.25$ \\
\hline \multicolumn{7}{|c|}{$\%$ homes built pre-1950 } \\
\hline$<5$ & - & & - & & - & \\
\hline$\geq 5$ to $<20$ & 1.45 & $1.17-1.80$ & 4.93 & $3.02-8.06$ & 4.08 & $2.24-7.43$ \\
\hline$\geq 20$ & 1.48 & $1.13-1.92$ & 9.93 & $5.97-16.50$ & 6.58 & $3.50-12.35$ \\
\hline \multicolumn{7}{|c|}{$\%$ homes built pre-1970 } \\
\hline$<15$ & - & & - & & - & \\
\hline$\geq 15$ to $<35$ & 1.58 & $1.25-2.00$ & 2.22 & $1.45-3.40$ & 1.72 & $1.02-2.91$ \\
\hline$\geq 35$ & 1.78 & $1.32-2.42$ & 5.33 & $3.35-8.49$ & 3.94 & $2.21-7.04$ \\
\hline \multicolumn{7}{|c|}{$\%$ renter occupied } \\
\hline$<10$ & - & & - & & - & \\
\hline$\geq 10$ to $<55$ & 1.06 & $0.84-1.34$ & 1.41 & $0.96-2.09$ & 1.50 & $0.86-2.61$ \\
\hline$\geq 55$ & 1.40 & $1.08-1.82$ & 2.97 & $2.00-4.42$ & 4.02 & $2.31-7.00$ \\
\hline \multicolumn{7}{|c|}{$\%$ vacant housing } \\
\hline$<5$ & - & & - & & - & \\
\hline$\geq 5$ to $<10$ & 0.85 & $0.69-1.04$ & 1.42 & $1.04-1.95$ & 1.81 & $1.16-2.82$ \\
\hline$\geq 10$ & 1.22 & $0.96-1.56$ & 2.54 & $1.82-3.55$ & 3.58 & $2.27-5.66$ \\
\hline \multicolumn{7}{|c|}{ Primary water source ${ }^{d}$} \\
\hline Groundwater & - & & - & & - & \\
\hline Surface water & 0.77 & $0.60-1.00$ & 0.80 & $0.56-1.15$ & 0.90 & $0.56-1.45$ \\
\hline
\end{tabular}

Missing values were excluded from analysis

Values in italics are statistically significantly at a significance level of $5 \%$

Ecological study design at the census tract level

a Age- and sex-adjusted incidence greater or equal to 2 cases per 100,000 persons

b Significant cluster with $50 \%$ of population at risk, relative risk greater or equal to 2 cases per 100,000 persons

c Exposed census tracts defined as RR $>2$ and reliability score $>0.5$

${ }^{d}$ Population-weighted centroids of census tracts were joined with public water system polygons 
Table 3 Adjusted odds ratios and $95 \%$ confidence intervals of macro-level demographic and environmental factors and increased odds of legionellosis occurrence

\begin{tabular}{|c|c|c|c|c|}
\hline & \multicolumn{2}{|c|}{ Model $A^{a}$} & \multicolumn{2}{|c|}{ Model $B^{b}$} \\
\hline & OR & $95 \% \mathrm{Cl}$ & OR & $95 \% \mathrm{Cl}$ \\
\hline \multicolumn{5}{|c|}{$\% \geq 65$ years of age } \\
\hline$<10$ & - & & - & \\
\hline$\geq 10$ to $<15$ & 0.62 & $0.40-0.97$ & 0.65 & $0.41-1.03$ \\
\hline$\geq 15$ & 0.38 & $0.22-0.67$ & 0.38 & $0.21-0.70$ \\
\hline \multicolumn{5}{|c|}{$\%$ non-white race } \\
\hline$<10$ & - & & - & \\
\hline$\geq 10$ to $<50$ & 0.85 & $0.49-1.48$ & 0.82 & $0.45-1.51$ \\
\hline$\geq 50$ & 1.68 & $0.92-3.07$ & 1.27 & $0.61-2.65$ \\
\hline \multicolumn{5}{|c|}{$\%$ hispanic ethnicity } \\
\hline$<5$ & - & & - & \\
\hline$\geq 5$ to $<20$ & 0.46 & $0.28-0.74$ & 0.46 & $0.28-0.75$ \\
\hline$\geq 20$ & 0.10 & $0.05-0.19$ & 0.10 & $0.05-0.21$ \\
\hline \multicolumn{5}{|l|}{$\%$ poverty } \\
\hline$<5$ & - & & - & \\
\hline$\geq 5$ to $<15$ & 1.52 & $0.91-2.52$ & 1.39 & $0.82-2.33$ \\
\hline$\geq 15$ & 7.21 & $4.04-12.86$ & 6.26 & $3.46-11.34$ \\
\hline \multicolumn{5}{|c|}{$\%$ less or some high school } \\
\hline$<5$ & - & & - & \\
\hline$\geq 5$ to $<10$ & & & 1.37 & $0.78-2.39$ \\
\hline$\geq 10$ & & & 1.25 & $0.67-2.32$ \\
\hline \multicolumn{5}{|c|}{$\%$ homes built pre-1950 } \\
\hline$<5$ & - & & - & \\
\hline$\geq 5$ to $<20$ & 4.63 & $2.43-8.82$ & 4.52 & $2.37-8.65$ \\
\hline$\geq 20$ & 5.69 & $2.82-11.50$ & 5.38 & $2.66-10.91$ \\
\hline \multicolumn{5}{|c|}{$\%$ renter occupied } \\
\hline$<10$ & - & & - & \\
\hline$\geq 10$ to $<55$ & & & 0.99 & $0.51-1.95$ \\
\hline$\geq 55$ & & & 1.28 & $0.54-3.02$ \\
\hline \multicolumn{5}{|c|}{$\%$ vacant housing } \\
\hline$<5$ & - & & - & \\
\hline$\geq 5$ to $<10$ & & & 1.19 & $0.70-2.03$ \\
\hline$\geq 10$ & & & 1.49 & $0.81-2.74$ \\
\hline \multicolumn{5}{|c|}{ Primary water source ${ }^{c}$} \\
\hline Groundwater & - & & - & \\
\hline Surface water & & & 0.70 & $0.41-1.22$ \\
\hline
\end{tabular}

Missing values were excluded from analysis

Values in italics are statistically significantly at a significance level of $5 \%$

Ecological study design at the census tract level

a Multivariate stepwise logistic regression model

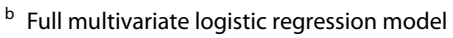

c Population-weighted centroids of census tracts were joined with public water system polygons

\section{Study results and literature}

As the percent of persons aged $\geq 65$ years increases from 10 to $14 \%$ to greater than $15 \%$, the negative association with legionellosis clustering becomes stronger.
Since people 50 years of age or older are known to be at increased risk for acquiring legionellosis [1], further research is required to determine why older age was a protective factor in this study. After controlling for other factors, census tracts with a $\geq 5$ to $<20 \%$ Hispanic population and $\geq 20 \%$ Hispanic population were 54 and $90 \%$ times less likely, respectively, to be classified as high-risk for legionellosis clustering. As with older age, Hispanic ethnicity was less likely to be associated with increased incidence or clustering or legionellosis. A recently published study that assessed race/ethnicity and Legionnaires' Disease incidence in New York (2002-2011) had similar findings; the average incidence per year for nonHispanic blacks was significantly higher than that for Hispanics [10]. Census tracts with more than $50 \%$ nonwhite populations were significantly more likely to be associated with legionellosis incidence and clustering, although, this effect was not seen while controlling for other factors, such as poverty. Associations with legionellosis and race were therefore largely driven by confounding from poverty, which was anticipated by a moderate correlation between the two continuous variables, and as suggested by previous research [15].

In both multivariate models, poverty level remained the strongest risk factor for legionellosis. The positive association between poverty level and legionellosis clustering strengthened as other factors, such as ethnicity and race were controlled for. The data show a distinct gradient in risk as the percentage of poverty level increases. Overall, incidence of Legionnaires' disease in the city of New York increased 230\% from 2002 to 2009 and followed a socioeconomic gradient, with highest incidence occurring in the highest poverty areas $[10,26]$. Among patients with community-acquired cases, the probability of working in transportation, repair, protective services, cleaning, or construction was significantly higher for those with Legionnaires' disease than for the general working population. Although socioeconomic status may be linked with risk factors for legionellosis (e.g., smoking, travel, underlying illness), a direct association between socioeconomic status and the risk of legionellosis has not been identified [34]. Factors related to socioeconomic status, recognized as an important determinant of certain chronic conditions, has not been extensively evaluated for its role in the incidence of infectious diseases. Incomplete information in case reports regarding individual level socioeconomic factors in many U.S. disease surveillance systems reduces the usefulness of surveillance data for these determinants. To our knowledge, no prior studies using group level data investigated the relationship between socioeconomic factors and legionellosis.

In our study, census tracts with higher proportions of rented (vs. owned) housing units were strongly associated 
with legionellosis incidence and clustering, although, this effect was not seen while controlling for other factors, such as poverty and pre-1950 housing. According to the American Housing Survey, rental units are more likely to be structurally inadequate than owner occupied units [35]. Given that older housing is also more likely to be inadequate, more than $13 \%$ of rentals built before 1960 have some structural deficiencies [36]. Older housing stock was also strongly associated with legionellosis clustering and areas of high occurrence. These effects appear to have a dose-response such that effect estimates were stronger for census tracts from the moderate to highest category of older housing for both pre-1950 or pre-1970, and were more elevate for older pre-1950 versus pre1970 housing stock. Older housing stock issues may be two-fold at both the individual and area-based level. Not only are older homes at risk of deteriorating plumbing systems leading to poor water quality, census tracts with older housing stock may also have an aging community water infrastructure providing homes with poorer water quality. Even when controlling for poverty and source of water, pre-1950 housing was strongly associated with legionellosis occurrence. Transmission of legionellosis has been reported to occur in private homes, yet as most outbreak investigations are focused on public buildings, outbreaks are more easily associated with large public buildings as compared with private residences [25]. There is a need for investigations to address how individuals acquire Legionella from their home, especially as these residential homes and infrastructures continue to age [37-39].

Public water distribution systems have been shown to play a role in the transmission of Legionella including potential contamination of the plumbing systems of buildings $[40,41]$. Since surface water is exposed to the environment, it was hypothesized that Legionella may be found at higher concentrations in census tracts served by these systems. Therefore, census tracts served by surface water sources may be at an increased risk of legionellosis occurrence compared with groundwater sources. However, no statistically significant associations with drinking water source water were found.

Pairwise correlations between population-level factors were not as strongly correlated as we predicted, since macro-environment factors are often predictive of one another (e.g. poverty and race, older housing stock, education levels). Area-based measures may be inherently collinear which may make multivariate analyses problematic. Condition indices were well below the cutoff of 30 and stepwise regression appears to have removed the more highly correlated factors. Future studies are needed to explore the exact mechanisms by which these risk factors may influence disease.

\section{Limitations}

Our study has inherent limitations based on its ecological study design. The associations identified between legionellosis and risk factors were measured at the census tract level and cannot be used to make individual inferences-this would result in biased interpretations known as ecological fallacy. Our cluster detection methods are limited to use of home address only; which could potentially result in exposure misclassification. This study did not have the capability to detect work-related or travelrelated clusters. A similar study conducted in New York City found that certain occupations might be associated with increased risk for community-acquired legionellosis [10]. We recommend that in the future, public health investigations collect work addresses during patient interviews and medical record reviews to enable prospective cluster detection surveillance to identify workrelated disease clusters. Due to limited data availability at the census tract level additional risk factors such as comorbidities and smoking were not explored and future research should include these important factors.

\section{Conclusion}

Along with well-established risk factors (e.g., older age, sex, immunocompromised), emerging variables such as socioeconomic and environmental factors may be significant indicators for legionellosis risk at the census tract level. The findings from this study are particularly important because they create an opportunity for public health authorities to better target communication for disease prevention. While geographic patterns alone are insufficient to conclude that these factors are causally related to the risk of legionellosis, these methods can be regarded as a first step approach in the evaluation of census tract level factors and the risk of legionellosis.

\section{Author's contributions}

JAG and KMR made substantial contributions to conception and design, acquisition of data, and analysis and interpretation of data; all authors contributed to drafting and revising of the work. All authors read and approved the final manuscript.

\section{Author details \\ 1 Environmental and Occupational Health Surveillance Program, New Jersey Department of Health, Trenton, NJ, USA. ${ }^{2}$ Communicable Disease Service, New Jersey Department of Health, Trenton, NJ, USA. ${ }^{3}$ Council of State and Ter- ritorial Epidemiologists Applied Epidemiology Fellowship, Atlanta, GA, USA.}

\section{Acknowledgements \\ None.}

Competing interests

The authors declare they have no competing interests.

\section{Availability of data and materials}

The case data used in this study are not publicly available to protect the Personal Health Identifiers of study participants but are available to investigators who obtain approval for human subjects' research for use of the data by the Rowan University Institutional Review board which serves as the New Jersey 
Department of Health (NJDOH) IRB of record. Additional information can be obtained by the NJDOH's Ethics Liaison.

\section{Consent for publication}

Not applicable.

\section{Ethics approval and consent to participate}

State of New Jersey Department of Health Institutional Review Board which was transferred to Rowan University Institutional Review Board which now serves as the IRB of record for this study has approved this study for human subjects'research.

\section{Funding}

This study/report was supported in part by an appointment to the Applied Epidemiology Fellowship Program administered by the Council of State and Territorial Epidemiologists (CSTE) and funded by the Centers for Disease Control and Prevention [8] Cooperative Agreement Number 1U38OT000143-05.

\section{Publisher's Note}

Springer Nature remains neutral with regard to jurisdictional claims in published maps and institutional affiliations.

Received: 24 August 2017 Accepted: 25 November 2017

Published online: 02 December 2017

\section{References}

1. World Health Organization. Legionella and the prevention of legionellosis, Bartram J et al., editors. 2007.

2. Dooling $\mathrm{KL}$, et al. Active bacterial core surveillance for legionellosis_United States, 2011-2013. MMWR Morb Mortal Wkly Rep. 2015;64(42):1190-3.

3. Marston BJ, et al. Incidence of community-acquired pneumonia requiring hospitalization. Results of a population-based active surveillance Study in Ohio. The Community-Based Pneumonia Incidence Study Group. Arch Intern Med. 1997;157(15):1709-18.

4. Government of Canada. Reported cases from 1924 to 2015 in Canadanotifiable diseases online. Legionellosis. http://diseases.canada.ca/notifiable/charts?c=pl. Accessed 15 Sept 2017.

5. Garrison LE, et al. Vital signs: deficiencies in environmental control identified in outbreaks of Legionnaires' disease-North America, 2000-2014. MMWR Morb Mortal Wkly Rep. 2016;65(22):576-84.

6. ECDC, European Centre for Disease Prevention and Control. Legionnaires's disease-annual epidemiological report for 2015. [Internet]. Stockholm; 2016.

7. Parr A, Whitney EA, Berkelman RL. Legionellosis on the rise: a review of guidelines for prevention in the United States. J Public Health Manag Pract. 2015;21(5):E17-26.

8. CDC. Legionellosis-United States, 2000-2009. MMWR Morb Mortal Wkly Rep. 2011;60(32):1083-6.

9. Neil K, Berkelman R. Increasing incidence of legionellosis in the United States, 1990-2005: changing epidemiologic trends. Clin Infect Dis. 2008;47(5):591-9.

10. Farnham A, et al. Legionnaires' disease incidence and risk factors, New York, New York, USA, 2002-2011. Emerg Infect Dis. 2014;20(11):1795-802.

11. van Heijnsbergen $E$, et al. Confirmed and potential sources of legionella reviewed. Environ Sci Technol. 2015;49(8):4797-815.

12. Hicks $L A$, et al. Increased rainfall is associated with increased risk for legionellosis. Epidemiol Infect. 2007;135(5):811-7.

13. Den Boer JW, et al. Results from the National Legionella Outbreak Detection Program, the Netherlands, 2002-2012. Emerg Infect Dis. 2015;21(7):1167-73.

14. Bhopal RS, Fallon RJ. Variation in time and space of non-outbreak Legionnaires' disease in Scotland. Epidemiol Infect. 1991;106(1):45-61.

15. Diez-Roux AV, et al. Area characteristics and individual-level socioeconomic position indicators in three population-based epidemiologic studies. Ann Epidemiol. 2001;11(6):395-405.
16. Conza L, et al. Meteorological factors and risk of community-acquired Legionnaires' disease in Switzerland: an epidemiological study. BMJ Open. 2013;3(3):e002428.

17. Gleason JA, et al. Under the weather: legionellosis and meteorological factors. EcoHealth. 2016;13(2):293-302.

18. Huang SW, et al. Legionella prevalence in wastewater treatment plants of Taiwan. Water Sci Technol. 2009;60(5):1303-10.

19. Cohn PD, et al. Community outbreak of legionellosis and an environmental investigation into a community water system. Epidemiol Infect. 2015;143(6):1322-31

20. Odoi A, et al. Determinants of the geographical distribution of endemic giardiasis in Ontario, Canada: a spatial modelling approach. Epidemiol Infect. 2004;132(5):967-76.

21. Younus $M$, et al. The role of neighborhood level socioeconomic characteristics in Salmonella infections in Michigan (1997-2007): assessment using geographic information system. Int J Health Geogr. 2007;6:56.

22. Green CG, Krause DO, Wylie JL. Spatial analysis of campylobacter infection in the Canadian province of Manitoba. Int J Health Geogr. 2006;5:2.

23. Rudbeck M, et al. Geographical variation of sporadic Legionnaires' disease analysed in a grid model. Epidemiol Infect. 2010;138(1):9-14.

24. Sansom P, et al. A case-association cluster detection and visualisation tool with an application to Legionnaires' disease. Stat Med. 2013;32(20):3522-38.

25. Prussin AJ, Schwake DO, Marr LC. Ten questions concerning the aerosolization and transmission of Legionella in the built environment. Build Environ. 2017;123:684-95.

26. Varga $C$, et al. Evaluating area-level spatial clustering of Salmonella Enteritidis infections and their socioeconomic determinants in the greater Toronto area, Ontario, Canada (2007-2009): a retrospective populationbased ecological study. BMC Public Health. 2013;13:1078.

27. Kulldorff M. SaTScan ${ }^{\text {TM }} .2015$.

28. Chen J, et al. Geovisual analytics to enhance spatial scan statistic interpretation: an analysis of U.S. cervical cancer mortality. Int J Health Geogr. 2008;7:57.

29. Han J, et al. Using Gini coefficient to determining optimal cluster reporting sizes for spatial scan statistics. Int J Health Geogr. 2016;15(1):27.

30. Wang $\mathrm{H}$, et al. Effect of disinfectant, water age, and pipe material on occurrence and persistence of Legionella, mycobacteria, Pseudomonas aeruginosa, and two amoebas. Environ Sci Technol. 2012;46(21):11566-74.

31. Kulldorff M. Bernoulli, discrete Poisson and continuous Poisson models: a spatial scan statistic. In: Communications in statistics: theory and methods. 1997. p. 1481-96.

32. Roth RE, Woodruff AW, Johnson ZF. Value-by-alpha maps: an alternative technique to the cartogram. Cartogr J. 2010;47(2):130-40.

33. CDC. Legionellosis_United States, 2000-2009. MMWR. 2011:60(32):1083-6.

34. Evans AS, Brachman PS. Bacterial infections of humans: epidemiology and control. 3rd ed. New York: Plenum Publishing Corporation; 1998.

35. Joint Center for Housing Studies. The State of the Nation's Housing. Joint Center for Housing Studies, editor. Harvard University: Cambridge, Mass; 1997.

36. Joint Center for Housing Studies. America's rental housing evolving markets and needs. Cambridge: Harvard University; 2013.

37. Muder RR, Yu VL, Woo AH. Mode of transmission of Legionella pneumophila. A critical review. Arch Intern Med. 1986;146(8):1607-12.

38. Fields BS, Benson RF, Besser RE. Legionella and Legionnaires' disease: 25 years of investigation. Clin Microbiol Rev. 2002;15(3):506-26.

39. Stout JE, Yu VL, Muraca P. Legionnaires' disease acquired within the homes of two patients. Link to the home water supply. JAMA. 1987;257(9):1215-7.

40. Lin YS, et al. Disinfection of water distribution systems for Legionella. Semin Respir Infect. 1998;13(2):147-59.

41. States SJ, et al. Survival and multiplication of Legionella pneumophila in municipal drinking water systems. Appl Environ Microbiol. 1987;53(5):979-86. 